\title{
REVIEW
}

\section{Progress in studies of sex determination mechanisms and sex control techniques in Cynoglossus semilaevis (half-smooth tongue sole)}

\author{
Qian ZHOU ${ }^{1,2}$, Songlin CHEN (ه) $)^{1,2}$ \\ 1 Key Laboratory for Sustainable Development of Marine Fisheries of Ministry of Agriculture, Yellow Sea Fisheries Research Institute, \\ Chinese Academy of Fishery Sciences, Qingdao 266071, China \\ 2 Laboratory for Marine Fisheries Science and Food Production Processes, Qingdao National Laboratory for Marine Science and \\ Technology, Qingdao 266373, China
}

\begin{abstract}
The Cynoglossus semilaevis (half-smooth tongue sole) is a marine flatfish of great commercial value for fisheries and aquaculture in China. It has a female heterogametic sex determination system (ZW/ZZ) and environmental factors can induce sex-reversal of females to phenotypic males, suggesting that it is a promising model for the study of sex determination mechanisms. Additionally, females grow much faster than males and it is feasible to improve the aquaculture production through sex control techniques. This paper reviews the progress in research on sex determination mechanisms research in our laboratory. We have completed whole-genome sequencing and revealed the genome organization and sex chromosome evolution of $C$. semilaevis. A putative male determining gene $d m r t 1$ was identified and DNA methylation was verified as having a crucial role in the sex reversal process. Genetic maps and sex-specific biomarkers have been used in a marker-assisted selection breeding program and for differentiation of the fish sex. Development and improvement of sex control technologies, including artificial gynogenesis and production of breeding fry with high proportion of females, is also reviewed. These research advances have provided insight into the regulation of sex determination and enabled efficient sex management in artificial culturing of $C$. semilaevis.
\end{abstract}

Keywords Cynoglossus semilaevis, sex determination, sex control technique

\section{Introduction}

Aquaculture of various finfish species is rapidly growing worldwide, which provides sustainable and important food sources for human consumption. Cynoglossus semilaevis (half-smooth tongue sole) is a large marine flatfish species that is widely distributed in Chinese coastal waters (Fig. 1). It is popular and well-known for its high nutritional and culinary value. As the first Soleidae fish species to be artificially cultivated, the sustainable farming of C. semilaevis has been successful in both industrial-scale hatcheries and pond culture practices. China is the largest producer and consumer of $C$. semilaevis with an annual farmed production valued at about 1.5 billion $\mathrm{CNY}^{[1]}$.

C. semilaevis exhibits significant sexual dimorphism. Females grow faster and the final body length and weight

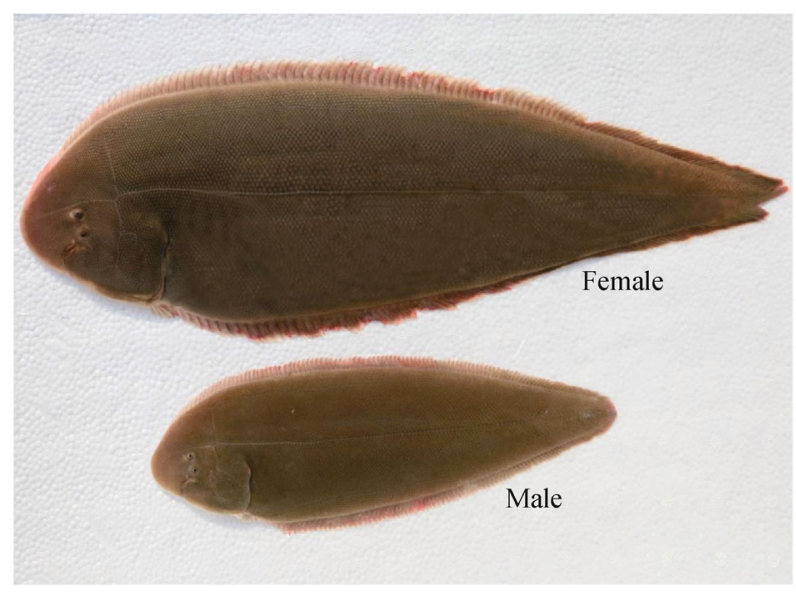

Fig. 1 Two year old female and male Cynoglossus semilaevis (half-smooth tongue sole) 
are two to four times that of males ${ }^{[2,3]}$. Consequently, females have higher productivity and economic value in aquaculture than males. Increasing the proportion of female fish to a high level has important commercial implications and is one of the most significant challenges for $C$. semilaevis culturing practice. To achieve this purpose, both fundamental biological understanding of sex determination mechanisms and development of practical sex control techniques are of great importance.

In recent decades, numerous studies have brought a boom in the understanding of the molecular and biological mechanisms in this species. C. semilaevis has complicated and special sex determining mechanisms, which are governed by both genetic and environmental factors. Karyotype analysis has revealed that $C$. semilaevis contains a total of 42 chromosomes $(2 n=42 \mathrm{t}, \mathrm{NF}=$ $42)^{[4]}$, including 20 euchromosome pairs and a pair of sex chromosomes ( $\mathrm{Z}$ and $\mathrm{W})$. The genetic sex determining mechanism of $C$. semilaevis was verified to be female heterogametic with ZW chromosomes whereas males have $\mathrm{ZZ}$ chromosomes ${ }^{[3,4]}$. In addition to the genetic inheritance of sex chromosomes, environmental factors, such as temperature, can also make a great contribution to the sexual fate of $C$. semilaevis. It has been observed that under normal culturing condition $\left(22^{\circ} \mathrm{C}\right), 14 \%$ of genetic females (with ZW sexual chromosomes) are sex-reversed to phenotypic males (i.e., sex reversed pseudo-males) ${ }^{[5]}$. Furthermore, the sex reversal rate increased to $73 \%$, when the rearing temperature was set to $28^{\circ} \mathrm{C}$ during a sensitive developmental period early in life ${ }^{[5]}$. Also, the ability to undergo sex reversal is heritable. The pseudo-males are fertile and can mate with normal females to produce viable offspring, which exhibit a sex reversal rate as high as $94 \%$, even under normal cultivating temperature $\left(22^{\circ} \mathrm{C}\right)^{[5]}$. All these features indicate that the $C$. semilaevis is an excellent model to investigate sex-determining mechanisms in fish. The interpretation of molecular mechanism underlying the sexual differentiation and reversal will also facilitate the genetic engineering of sex control and genome selection breeding with the aim of increasing production and quality in aquaculture.

Through years of research, we have made significant progress in studies of the sex determination and the development of sex control techniques. As the first genome-sequenced flatfish, the availability of the C. semilaevis genome resource has provided new knowledge about the genetics of sex determination and other physiological processes in C. semilaevis. Combined with transcriptomic and epigenetic analysis, we have discovered the putative male-determining gene dmrtl and methylation regulation in the sex determination. A variety of genetic linkage maps have been constructed, providing a powerful tool for research on genome evolution and brood stock enhancement projects using selective breeding. Highly sensitive sex-specific biomarkers have been identified and successfully applied to differentiation of sex in C. semilaevis and have promoted the development of new technologies for sex control, such as artificial gynogenesis and breeding fry production with a high proportion of females. Such sex control techniques will be effective in increasing the proportion of female C. semilaevis, thus improving aquaculture productivity and profitability. Further refinement of these techniques and development of new techniques is needed and could enable biological and genetic engineering of the sex control in large-scale aquaculture.

\section{Genetic mechanism of sex determination}

\subsection{Genome sequencing and sex-determining gene}

In teleost, there are three types of sex determination mechanisms. One is genetic sex determination (GSD), meaning that sex is determined through sex chromosomes and sex determining genes. The second type is environmental sex determination (ESD), under which environmental factors, such as temperature, $\mathrm{pH}$ and light intensity can influence sex differentiation. The third type is genetic determination plus environmental effects (GSD + EE). There is a plastic interaction between genetic and environmental factors, making a combined action on the sexual fate of fish. It is known that $C$. semilaevis employs a female heterogametic sex determination system (ZW/ZZ). Surrounding environmental conditions, such as high temperature, can induce sex-reversal of female fish to phenotypic males. As a fish species with clear sex-specific difference in morphology, the mechanisms underlying the sex determination of $C$. semilaevis are an important and interesting scientific question. After years of effort, significant advances have been made in the study of sex determination mechanism of $C$. semilaevis.

An important foundation of genome analysis is the construction of bacterial artificial chromosome (BAC) libraries. BAC libraries provide a platform for the construction of physical maps and complete genome sequencing, which are powerful tools for large-scale gene discovery and elucidation of gene function and regulation. BAC libraries are also applied in map-based cloning of quantitative trait loci, molecular cytogenetics and genomic variation identification. To obtain the genomic sequence of $C$. semilaevis, two BAC libraries with three females and a high resolution BAC-based physical map of $C$. semilaevis were constructed ${ }^{[6]}$. A total of 55296 BAC clones were obtained, corresponding to 13.4 haploid genome equivalents. In the BAC library, it is estimated and validated that there is a $99 \%$ probability that each clone has a single-copy sequence ${ }^{[6]}$, indicating the library was established with high reliability and accuracy. Based on the BAC library, the assembly consisted of 1485 contigs with an N50 length of $664 \mathrm{~kb}$ and an estimated physical length of $797 \mathrm{Mb}$, equivalent to about 1.27 
coverage of the C. semilaevis genome ${ }^{[7]}$. The BAC-based physical map has been used for the integration of physical and genetic maps, fine-mapping of important genes and/or quantitative trait loci (QTL), and comparative and evolutionary genomics studies.

In recent years, development of next-generation sequencing technology has provided high-throughput and lowcost sequencing platforms, permitting research and interpretation of the sequenced organism from a systematic point of view. The whole-genome sequencing of C. semilaevis was completed in 2014 using the Illumina sequencing platform. To reveal the genetic basis of its sex determination, the genomic DNA of a female and a male C. semilaevis was sequenced. High quality shotgun reads were obtained with a sequencing depth of 121-fold for each fish. The genome size of $C$. semilaevis was estimated to be $477 \mathrm{Mb}$, with the scaffold N50 of $867 \mathrm{~kb}$ and an average GC content of $40.8 \%$ (Fig. 2) ${ }^{[5]}$. The previously established BAC libraries were used to overcome some difficulties in assembly of the short reads, such as repetitive sequences and gaps. A variety of sequence information and biomarkers in several ready-to-use physical map and genetic linkage maps were used to facilitate the genome assembly, including a BAC-based physical map ${ }^{[7]}$, microsatellite genetic maps ${ }^{[8-11]}$ and a high-resolution SNP genetic map. The microsatellite and SNP genetic markers were assigned to 22 linkage groups corresponding to the 20 autosomes and two sex chromosomes ( $\mathrm{Z}$ and $\mathrm{W})$. In the final assembly, a high proportion of the sequences (93.3\%) of $445 \mathrm{Mb}$ was consistently assigned and localized on 22 linkage groups, containing 19800 genes $(92.0 \%$ out of all predicted genes ${ }^{[5]}$. Based on the genome assembly, the final gene set of $C$. semilaevis contained 21516 genes, $99 \%$ of which were confirmed by homologous genes in other organisms or in the transcriptome sequencing. More than $94 \%$ of the predicted genes were functionally annotated in the Swiss-Prot database and GO database ${ }^{[5]}$. The phylogenetic relationship and divergence time were analyzed by comparing the genome of $C$. semilaevis with other teleost fishes using human and chicken genomes as

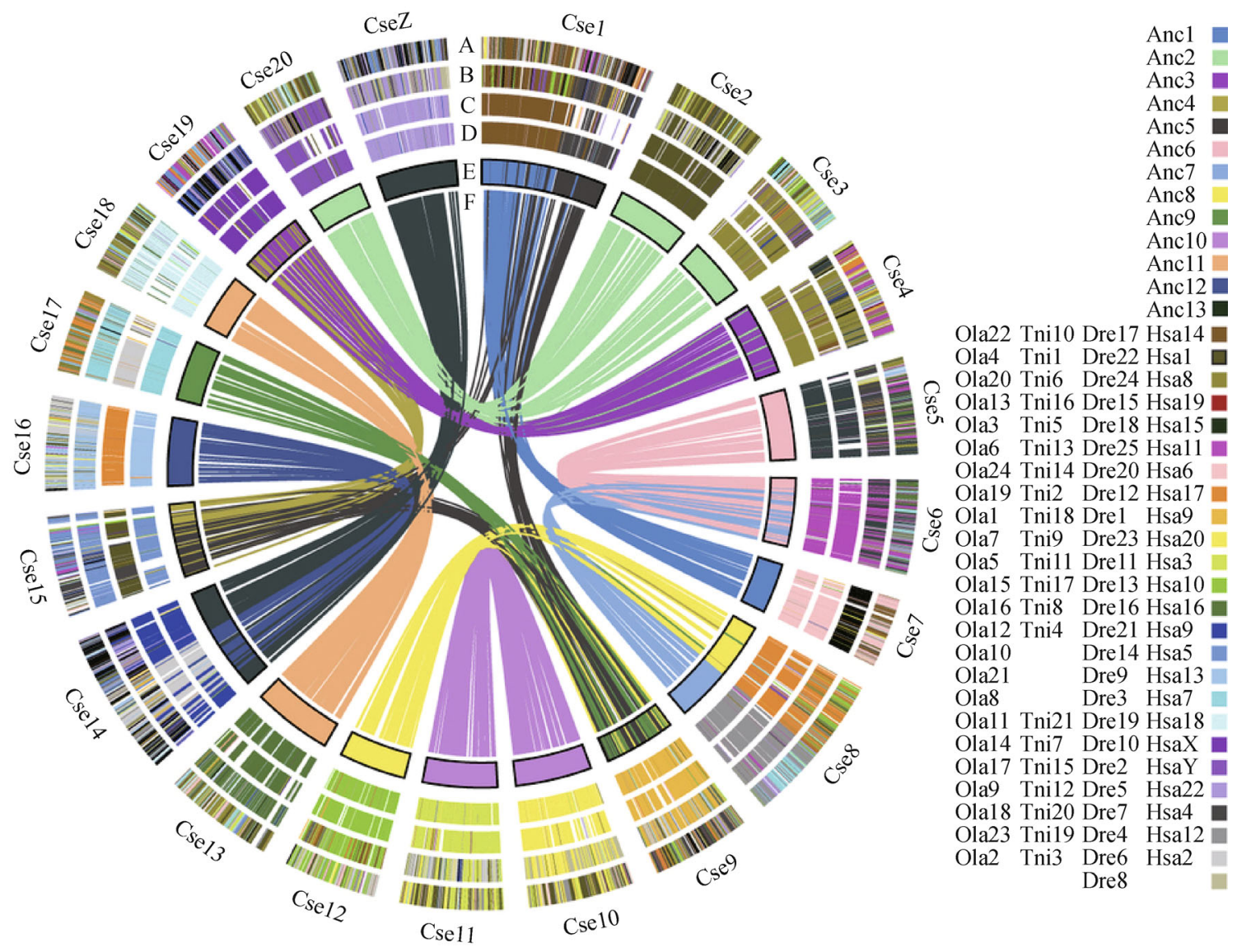

Fig. 2 Teleost genome evolution. Whole-genome duplication in Cynoglossus semilaevis and orthology in Oryzias latipes, Tetraodon nigroviridis, Daniorerio, and human genomes. The arcs of concentric circles represent each C. semilaevis chromosome (Cse1-Cse20 and CseZ). A-D, tongue sole chromosomes indicated with different colors according to the location of the orthologs in the human (Hsa), D. rerio (Dre), T. nigroviridis (Tni) and O. latipes (Ola) genomes. A $100 \mathrm{~kb}$ region around a gene is indicated in the same color; E, C. semilaevis chromosomes indicated by the corresponding ancestral chromosomes (Anc1-Anc13); F, each line joins duplicated genes at their respective positions (adapted from Chen et al. ${ }^{[5]}$ ). 
outgroups. It is estimated that flatfish diverged from other bony fish about 197 million years before the present.

The genomic sequences of both $\mathrm{W}$ and $\mathrm{Z}$ chromosomes were identified through comparison of the female and male assemblies, with scaffold length of 23.3 and $16.4 \mathrm{Mb}$, respectively. Notably, the $\mathrm{Z}$ chromosome sequence in C. semilaevis is the first full sequence of the $\mathrm{Z}$ chromosome outside of birds ${ }^{[5]}$. Phylogenetic and comparative analysis allowed identification of many important evolutionary events of the sex chromosomes and provided insights into its sex determining mechanisms ${ }^{[5]}$. The $C$. semilaevis $\mathrm{W}$ linked and Z-linked scaffolds were analyzed and compared with sex chromosomes of mammals and birds. This showed that the sex chromosomes of $C$. semilaevis have evolved from a pair of ancestral vertebrate autosomes and massive gene loss occurred in the early stage of sex chromosome formation. The age of sex chromosomes in C. semilaevis was determined to be about 30 million years, demonstrating that $C$. semilaevis has young sex chromosomes compared to those of mammals and birds which date back hundreds of millions of years. Therefore, there are still many intact genes in the non-recombining region of the $C$. semilaevis $\mathrm{W}$ chromosome ${ }^{[5]}$. Moreover, the sex chromosomes of $C$. semilaevis are highly conserved with those of chicken, which has a similar ZW sex chromosomal system ${ }^{[5]}$.

In C. semilaevis, sex determination operates through a Z-encoded mechanism that determines male development. A putative functional male sex-determining gene dmrt1 (double sex and mab-3 related transcription factor) was discovered on the $\mathrm{Z}$ chromosome. Dmrt1 was specifically expressed in male germ cells and pre-somatic cells of the undifferentiated gonad at the sex-determination stage and persisted at high levels during testis development ${ }^{[5]}$ (Fig. 3a, Fig. 3b). In ZW females, the promoter region of dmrt1 was in a methylated state, whereas in sex-reversed pseudo-males, demethylation patterns occur at the same loci, which activated its transcription (Fig. 3c). Through a dosage compensation approach, the expression of dmrtl in $\mathrm{ZW}$ pseudo-males is upregulated to a level that is normal for ZZ males. Additionally, as a transcription factor, the recombinant DMRT1 protein of C. semilaevis was able to regulate the expression of several sex related genes. It inhibited the expression of cytochrome P450, family 19 , subfamily A, polypeptide 1 (cyp19a) and winged helix fork-head transcription factor gene 2 (foxl2), but increased the expression level of $\operatorname{sox} 9 a$, implying its regulatory role in sex differentiation ${ }^{[12]}$. All these features raise the possibility that $d m r t 1$ is the critical gene that responds to environmental change and triggers the sex reversal cascade in C. semilaevis ${ }^{[5]}$.

\subsection{Epigenetic regulation and dosage compensation}

In addition to GSD, ESD interacts strongly with GSD mechanisms and influences the sex fate of $C$. semilaevis. (a)
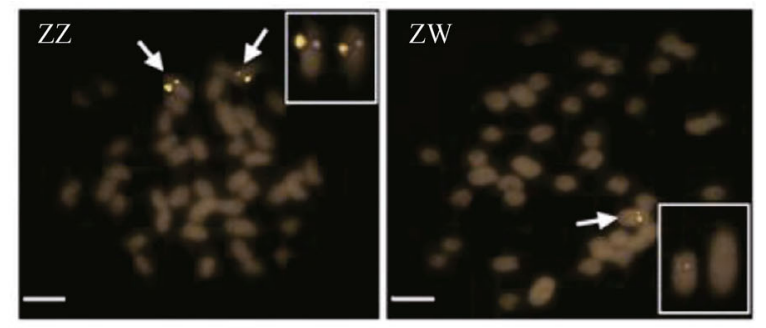

(b)

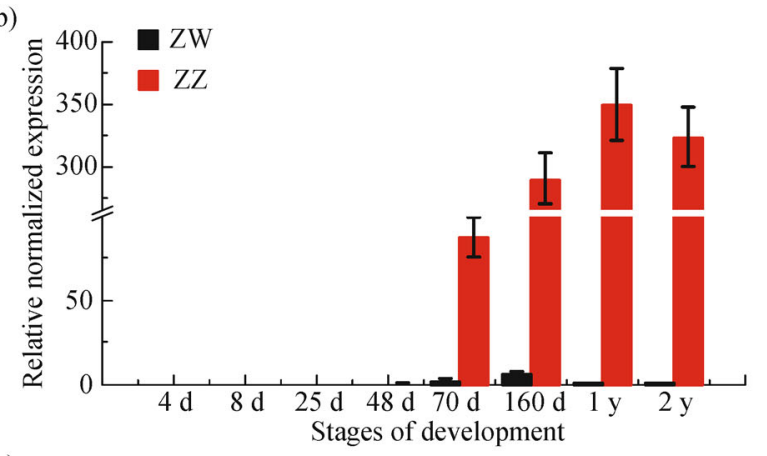

(c)

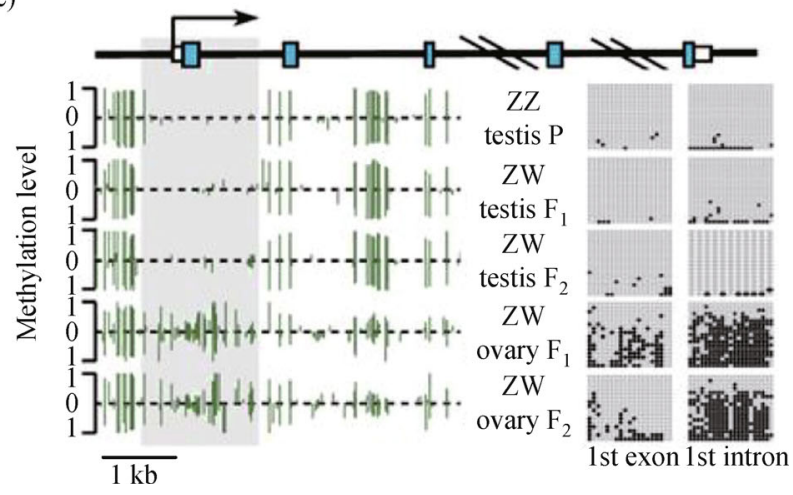

Fig. 3 Characterization of dmrt1 in half-smooth tongue sole. (a) Dmrt1 BAC FISH analysis of tongue sole chromosomes showing a double signal in males and a single signal in females; (b) RT-PCR analysis of dmrt1 during developmental stages in female (black bar) and male (red bar) tongue sole. The data are shown as the mean \pm s.e.m. $(n=3)$; (c) methylation status across the differentially methylated region (DMR) of $d m r t 1$ in the gonads of an adult WZ female, a ZZ male and a WZ female compared to male sex-reversed fish. The schematic diagram at the top shows the genomic structure of dmrt1 in tongue sole. Blue boxes, exons; White boxes, $3^{\prime}$ and 5' UTR regions; Black arrow, the direction of the $d_{m r t 1}$ gene from the transcriptional start site; Green line, the methylation level of each cytosine, identified on both DNA strand throughout the dmrt1 gene in female and male fish; Gray shadow, the DMR. Data are adapted from Chen et al. ${ }^{[5]}$.

However, the molecular biology of ESD remains poorly understood. To understand the regulation of ESD, we investigated the role that epigenetics (such as DNA methylation, which has been analyzed by bisulfite sequencing) plays in controlling the sex reversal process. Genome-wide profiling of methylome analysis was performed in C. semilaevis, showing that DNA methylation is crucial in the transition from GSD to ESD in the sex reversal process. Firstly, the methylation pattern of the 
pseudo-male fish is highly similar to that of the normal males. The epigenetically regulating genes expressed during the sexual reversal process are significantly enriched in those involved in known sex determination pathways. Secondly, this epigenetic modification can be inherited by the ZW offspring of pseudo-males, so that most of these genetically female offspring become phenotypic males without high temperature induction. As a result, the percentage of phenotypic males in the offspring of pseudo-males can reach as high as $95.5 \%{ }^{[13]}$.

Moreover, for animals with sex-determining chromosomes, it is critical to overcome dosage imbalance of the sex chromosomes after phenotypic sex reversal. Compared to normal males (ZZ) of $C$. semilaevis, the pseudo-males (ZW) have one more female-specific W chromosome and one less $\mathrm{Z}$ chromosome. Specific dosage compensation occurs in a unique $\mathrm{Z}$ chromosome region in pseudo-males, with gene expression level equal to that in normal male testis, enabling them to overcome insufficient gene expression relative to normal ZZ males. Methylation variable positions and genes involved in spermatogenesis are enriched in this region. Simultaneously, there is a divergence in gene expression on the $\mathrm{W}$ chromosome. Some genes maintain active expression in pseudo-males, which is proposed to function in the dosage compensation of the genes on the $\mathrm{Z}$ chromosome. However, some female specific genes, such as figla, were inhibited by methylation regulation, possibly because their expression would interfere with the normal male phenotype ${ }^{[13]}$.

\subsection{Sex related genes}

Sex determination is a complex polygenic trait in fish. In C. semilaevis, a number of similar sex-related genes were identified, suggesting a conservation of sex determination pathways. In addition to the putative male sex determinant gene dmrt1, there is an E3 ubiquitin ligase gene neurl3, which is present on the $\mathrm{Z}$ chromosome but absent from the $\mathrm{W}$ chromosome. It is highly expressed during spermatogenesis and $\mathrm{W}$-chromosome sperm that lack this gene do not develop in sex-reversed WZ pseudo-males, indicating that it is a potential male-beneficial gene in $C$. semilaevis.

Gene expression analysis has been widely employed to study the sex-related genes in C. semilaevis, revealing their temporal and spatial expression patterns. The transcription factors gene $\operatorname{sox} 9 a$ exhibited a higher expression in gonads of pseudo-males than that of normal females with a significant regulation during the period of sex differentiation, which was considered to have a close link with sex reversal, sex differentiation and cell differentiation of embryos and formation of spermatogenic cells ${ }^{[14]}$. Cytochrome P450-aromatase, the enzyme catalyzing conversion of androgens to estrogens, seems to be a critical enzyme for ovarian differentiation. Two P450 aromatases were found in C. semilaevis. P450aromA belongs to the gonadal P450arom subfamily, transcripts of which were highly abundant in ovaries, but lower in testis and not present in other tissues ${ }^{[15]}$. P450aromB belongs to the brain P450arom subfamilies, which shares $45.1 \%$ sequence similarity with $\mathrm{P} 450$ aromA in $C$. semilaevis and had a high expression level in the brain and gills but lower in gonads and skin. However, the P450aromB transcript was down-regulated in the brain of sex-reversed males after treatment with methyltestosterone or at high temperatures $^{[16]}$. Anti-Müllerian hormone $(\mathrm{amh})$ is a glycoprotein belonging to the transforming growth factor $\beta$ superfamily, which has a major role in reproductive development in vertebrates. In C. semilaevis, the expression level of the amh gene increased in the gonads of males and pseudo-male offspring, but did not change in females, indicating that the amh gene is required for sex reversal and during reproductive development ${ }^{[17]}$. Three homologous genes encoding growth arrest and DNA-damageinducible protein 45 gamma (gadd45g) were identified in C. semilaevis, among which, gadd45g1 may be necessary for sex differentiation in the early stage of gonad development, and both gadd45gl and gadd45g2 function in maintaining ovary development and the female phenotype of $C$. semilaevis ${ }^{[18]}$. The gadd45g3 gene is necessary for testes maturation and is involved in sex determination prior to gonadal differentiation ${ }^{[19]}$. The Wilms' tumor suppressor gene, $w t$, is another sex-related gene. Its expression in testes was significantly higher than that in ovaries and gonads of sex-reversed female fish. Among them, the lowest expression was found in the gonad of sex-reversed females ${ }^{[20]}$. The transcript of fushi tarazu factor-1 gene $(f t z-f 1)$ was distinctly expressed in the embryo rather than in larvae, which suggested that the $f t z-f 1$ gene may be involved in the organogenesis in C. semilaevis ${ }^{[21]}$. The ubiquitin-conjugating enzyme $(u b c 9)$ gene may also function in the sex reversal process because its transcript level was significantly higher in the temperature-treated females than the normal females and males $^{[22]}$.

\section{Sex-specific markers}

High quality genetic linkage maps provide a promising platform for marker-assisted selection breeding programs for commercially important traits and systematic genome searches for QTLs, relating to disease resistance, growth and sex-related traits. Tremendous advances have been made in the genetic map system and marker detection techniques. In recent years, considerable effort has been applied to genetic studies and a variety of genetic linkage maps of $C$. semilaevis have been built, which has significantly improved the pace and precision of fish genetic analysis.

For C. semilaevis, distinguishing female and male individuals and increasing the female ratio can improve the profitability of aquaculture. After years of research, a 
large number of genetic biomarkers, especially sex-specific molecular markers, have been identified and successfully applied to the determination of sex genotype. Furthermore, easy and powerful sex discrimination technology has been developed using viable markers, allowing quick and precise sex identification in C. semilaevis. As important genomic resources, the molecular genetic markers also have important application in the study of genetic diversity and population structure.

3.1 AFLP genetic linkage map and female-specific AFLP markers

A first generation genetic linkage map in C. semilaevis was established based on amplified fragment length polymorphism (AFLP) technology. The AFLP-based genetic map has a total length of $934.6 \mathrm{cM}$ and an average spacing of $8.4 \mathrm{cM}$. A total of 137 markers including 103 AFLP markers, 33 microsatellite markers, and one femalespecific DNA marker were mapped on 20-six linkage groups (LGs) ${ }^{[9]}$. This was the first genetic linkage map for C. semilaevis, and it delivered great potential applications in the development of marker-assisted selection breeding techniques.

Through validation tests in artificially propagated fish individuals, seven AFLP molecular markers were verified as specifically present in females, but absent in male C. semilaevis ${ }^{[23]}$. According to the flanking sequence of the marker, CseF382, an easy-to-use and quick PCR detection method was successfully developed for distinguishing the female (ZW) and male (ZZ) half-smooth C. semilaevis (Fig. 4a) ${ }^{[23]}$. This was the first genetic molecular marker that could successfully differentiate the sex of C. semilaevis, and it has been widely applied in differentiating male (ZZ) and female (ZW) individuals in culturing fish. However, as a dominant inheritance molecular marker, this AFLP marker cannot distinguish homozygotes from heterozygotes. Therefore, new and accurate co-dominant molecular markers are required, which can distinguish ZW females and WW superfemales.

\subsection{Microsatellite genetic linkage map and sex-specific SSR markers}

Microsatellites, or simple sequence repeats, represent codominant molecular genetic markers, which are abundantly present within genomes. They are short segments of DNA that have a repeated sequence. Due to a high level of polymorphism, a relatively small size and rapid detection protocols, microsatellite markers have been widely used in construction of genetic linkage maps, quantitative traits mapping and identification of genes or mutation responsible for a given trait for marker-assisted breeding programs.

Considerable efforts have been made to identify microsatellite markers and quite a number of polymorphic microsatellite markers have been characterized in C. semilaevis ${ }^{[9-11,25]}$. These markers have facilitated the evaluation of the genetic diversity, assessment of important quantitative traits and brood stock management of this species. In 2012, a high density microsatellite genetic linkage map, which is considered as the second-generation genetic map of C. semilaevis, was published (Fig. 5 ${ }^{[8]}$. Female and male $C$. semilaevis were separately used to build sex-specific maps, which were then integrated into a high quality consensus microsatellite genetic linkage map. On this map, a total of 1007 microsatellite markers and

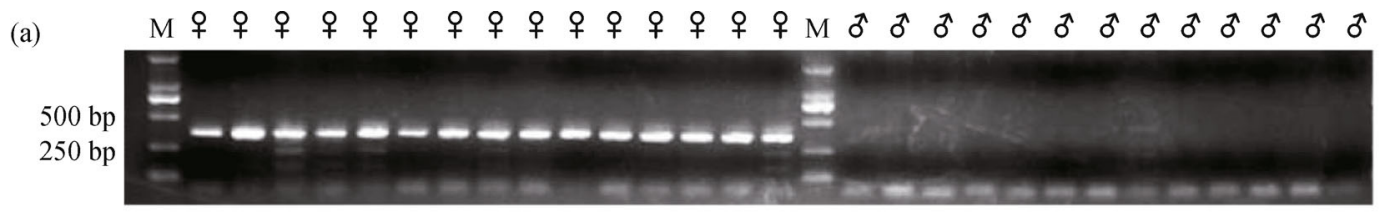

(b)

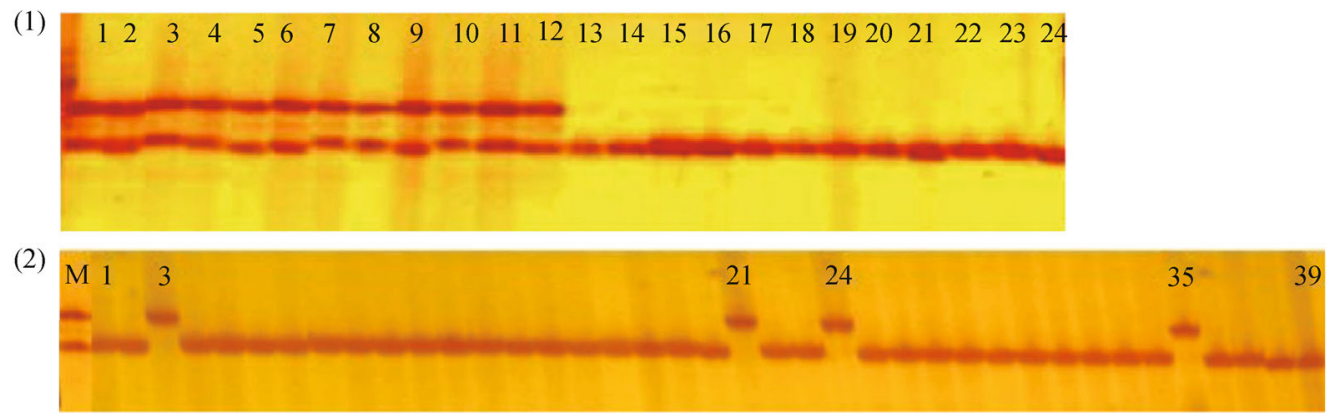

Fig. 4 Genetic sex identification of the half-smooth tongue sole using sex-specific markers. (a) AFLP genetic marker CseF382; (b) microsatellite genetic markers CseF-SSR1: (1) identification of females (ZW) and males (ZZ) (1-12, females; 13-24, males); (2) identification of males (ZZ) and super-females (WW) in mitogynogenetic embryos( 3, 21, 24, 35 are super-females; others are males) (adapted from Chen et al. ${ }^{[24]}$ ). 

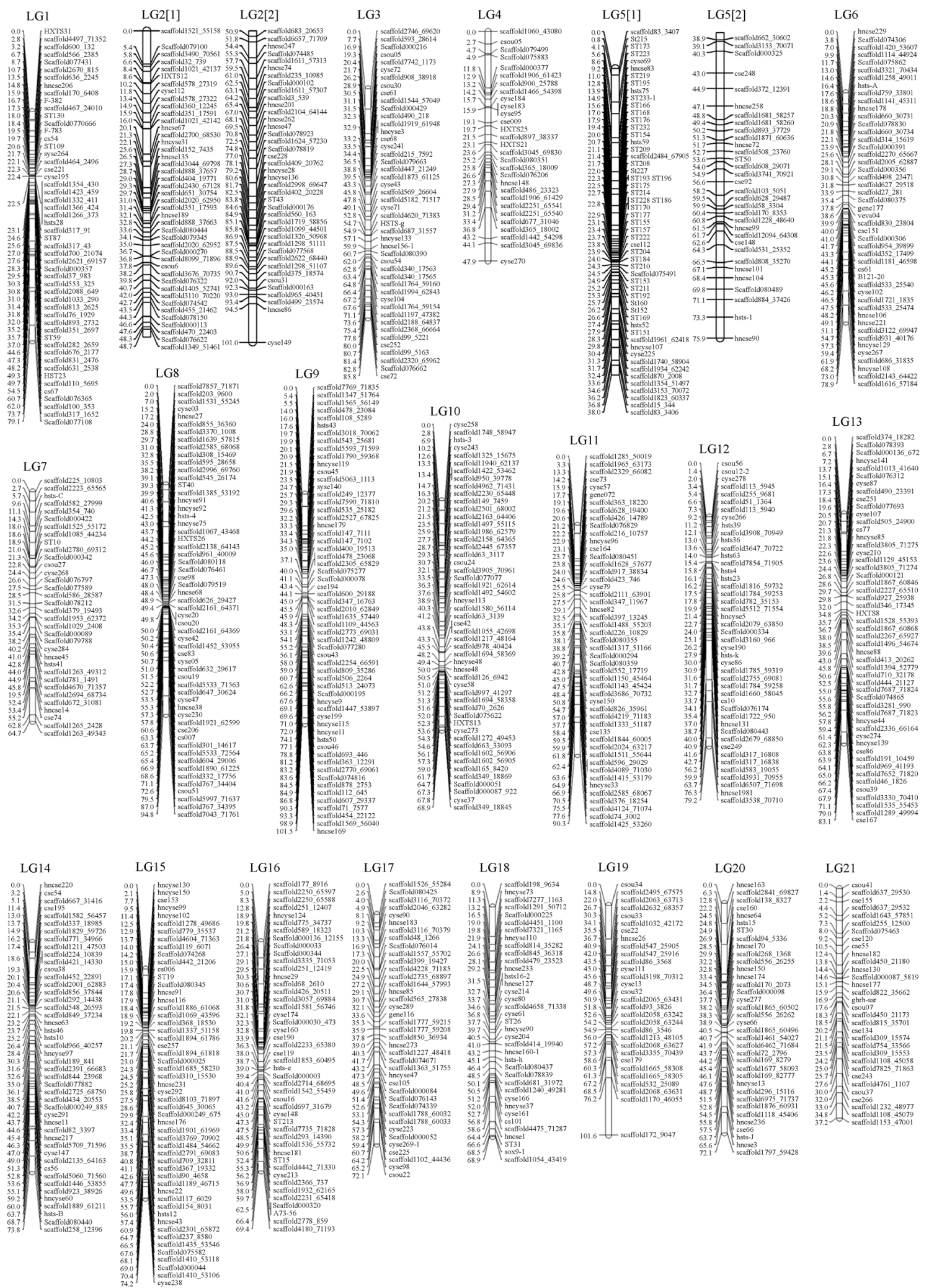

Fig. 5 Microsatellite linkage map for Cynoglossus semilaevis. The consensus genetic map comprises 1009 markers assigned to 21 linkage groups (LG1-LG21). Genetic distances in Kosambi centimorgans are listed on the left side of the linkage groups, and markers are listed on the right side of the linkage groups (adapted from Song et al. ${ }^{[8]}$ ). 
two SCAR markers located in 21 linkage groups were mapped, covering a total of $1624 \mathrm{cM}$ with an average interval of $1.67 \mathrm{cM}^{[8]}$. Of particular interest are 812 and 785 positions identified to be unique in female and male fish, respectively. For this map, a total of 159 sex-linked SSR markers were identified and five sex-linked microsatellite markers were validated as being associated with sex in a large number of individuals selected from different families. Notably, after large-scale validation in C. semilaevis population, the SSR marker CseF-SSR was verified to be highly effective in sex discrimination for C. semilaevis (Fig. 4b) ${ }^{[24]}$. Using this marker, the PCR amplification of the genomic DNA produced one DNA band of $206 \mathrm{bp}$ in ZZ males, two DNA bands of $206 \mathrm{bp}$ and $218 \mathrm{bp}$ in ZW females, and one DNA band of $218 \mathrm{bp}$ in WW super-females ${ }^{[24]}$. Currently, the CseF-SSR marker is used as the workhorse to differentiate the genetic female, male and super-female fish for the practical operation of sex determination. This sex-linked molecular marker is able to identify the genetic sex of ZW and WW of gynogenetic diploids of $C$. semilaevis, a critical technical prerequisite, providing a foundation for molecular sex control techniques.

\subsection{SNP genetic linkage map and SNP markers}

Recently, markers based on Single Nucleotide Polymorphisms (SNPs) have gained increasing popularity due to their abundance in the genomes and their amenability for highthroughput detection techniques. The SNP markers are excellent for studying complex genetic traits and for understanding the genomic evolution, as well as population genetics. The availability of whole genome sequences of C. semilaevis sole provided a foundation for large-scale comparative genomics research and SNP marker identification. Using genomic re-sequencing techniques, a high density consensus genetic linkage map was developed for a C. semilaevis family of 216 individuals. On this map, 12142 SNPs are assigned to 22 linkage groups, one more than the haploid number of chromosomes of $C$. semilae$v i s^{[5]}$. The average marker interval is $0.326 \mathrm{cM}$ and the total linkage distance is $1900.47 \mathrm{cM}$, which represents a high marker density that could not be achieved with other classes of genetic marker.

\section{Biological technology for sex control in C. semilaevis}

Female and male $C$. semilaevis have distinctly different growth rates and morphology. Therefore, a key aim in $C$. semilaevis aquaculture is the improvement of phenotypic female production with the desired growth traits to maximize profitability. Genetic engineering for sex control and monosex cultivation is a feasible and effective approach, and of potentially great benefit for both research and production.

\subsection{Artificial gynogenesis}

Gynogenesis is a powerful approach to producing a population of homozygotes, studying sex determination mechanisms and developing pure lineages in fish. In artificial gynogenesis, eggs are activated by heterogeneous or inactivated sperms, and the genetic information/ chromosomes of offspring are inherited solely from the mother. The induction process mainly involves two steps, the inactivated sperm stimulation and the diploidization of female chromosomes. There are two types of artificial gynogenesis: meiogynogenesis and mitogynogenesis. In meiogynogenesis, diploidization of the chromosome set is performed by blocking the extrusion of the second polar body. In mitogynogenesis, chromosome diploidization is performed by blocking the first cleavage. Theoretically, in mitogynogenetic embryos, almost all the loci are homozygous, suggesting it is a promising strategy for pure line establishment, monosex population production, genetic mapping and species preservation.

Since the $C$. semilaevis has a female heterogametic (ZW) sexual chromosome system, artificial gynogenetic induction makes it possible to generate normal males (ZZ) and super-females with WW chromosomes. When superfemales are available, all-female (ZW) populations can be produced through crossing the WW super-females with normal males $(\mathrm{ZZ})^{[3]}$. Therefore, artificial gynogenesis is a promising method to control the sex and produce all female progeny of $C$. semilaevis. Both meiogynogenesis and mitogynogenesis protocols have been developed for C. semilaevis ${ }^{[3,24]}$. Cryopreserved Lateolabrax japonicas (sea perch) sperm are inactivated by UV light and then used to trigger gynogenesis. Key parameters in chromosome diploidization treatment, including initiation time, intensity of pressure or temperature shock and treatment duration, were optimized and validated to be effective ${ }^{[3,24]}$. The gynogenetic haploid and diploid chromosome numbers were 21 and 42 , respectively ${ }^{[25]}$. In gynogenetic diploid, two huge WW chromosomes that had developed by $\mathrm{W}$ chromosome duplication were observed in some of the gynogenetic embryos (Fig. 6) ${ }^{[3]}$. In addition, the average percentage of homozygosity reached $80.5 \%$ in the mitogynogenetic diploid larvae. However, about $10 \%$ of genetic females (WW) in the heterogeneous gynogenetic larvae were observed, possibly because of a high death rate of WW super-females.

In summary, the discovery of WW super-female genotype clearly demonstrated that $C$. semilaevis has a ZW sex determination mechanism ${ }^{[24]}$. The artificial gynogenesis technology provides an important tool for the investigation of sex determination mechanism, breeding of all-female stock, isolation of the sex determining loci and sex manipulation in C. semilaevis. 

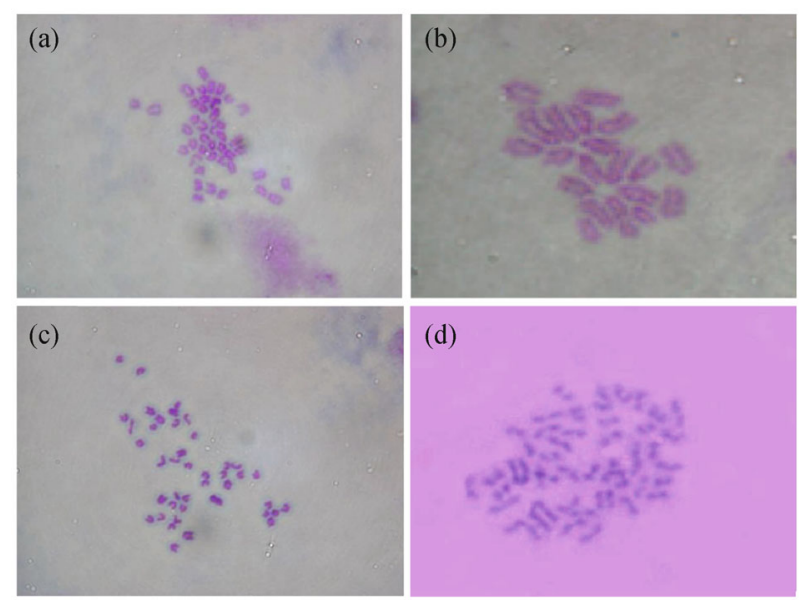

Fig. 6 Chromosome analysis of normal embryos and gynogenetic embryos. (a) Normal male tongue sole; (b) gynogenetic haploids; (c) gynogenetic diploids, showing a ZZ individual; (d) gynogenetic diploid embryos, showing a WW individual, two huge WW chromosomes were observed (arrowhead) (adapted from Chen et al. $\left.{ }^{[3]}\right)$.

More research is needed to further optimize technical parameters and improve the survival rate of the WW superfemale fry, followed by extension to ensure application in large-scale gynogenetic fry production.

\subsection{Breeding fry production with high proportion of females}

Although females grow fast and are thus the preferred gender for C. semilaevis aquaculture, only about $20 \%$ (ranging from $10 \%$ to $30 \%$ ) of breeding C. semilaevis fry are phenotypic females (while the theoretical percentage of genetic females is 50\%). The primary reason for this phenomenon is the special features of pseudo-males. Firstly, it has been observed that the proportion of phenotypic females in the $F_{1}$ generation from pseudomales is considerably lower than from normal males. Secondly, in the offspring of parental pseudo-males, sex reversal of genetic females to pseudo-males is common and reaches about 94\%. Therefore, the offspring of parental pseudo-males are dominantly males, which influence the overall production. Therefore, in the artificial propagation of $C$. semilaevis, if the pseudo-males can be isolated from the breeding population and only normal $\mathrm{ZZ}$ males are used as the male parent, the breeding yield of phenotypic females, as well as the overall fish production can be considerably improved.

Using the sex-specific biomarkers, the genetic sex of fish individuals can be precisely confirmed, regardless of their phenotypes. Therefore, sex-specific markers are needed for the development of breeding fry production with a high proportion of females. With this technique, the genetic males (ZZ) and pseudo-males (ZW) are separated and only

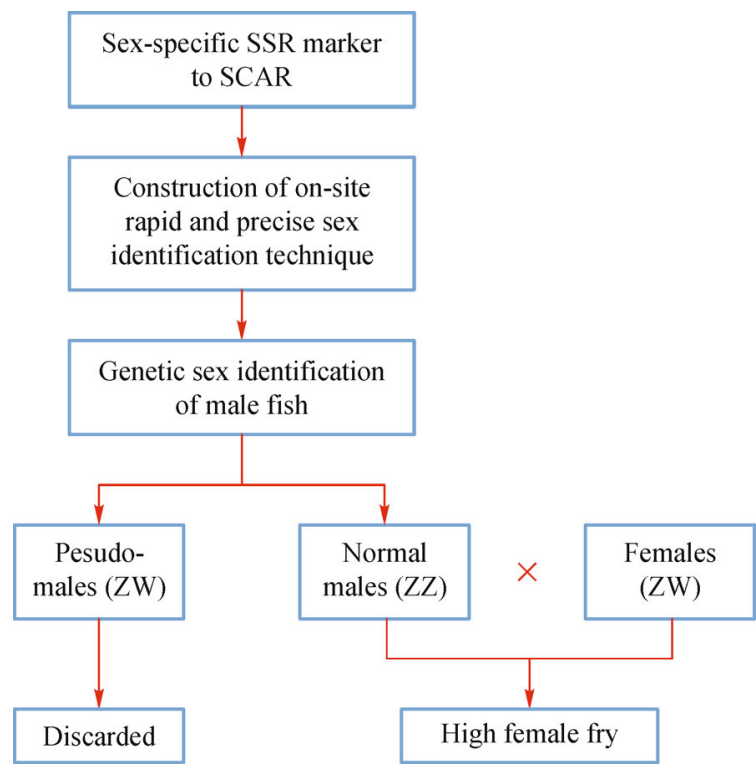

Fig. 7 Roadmap of the high female breeding technology. SSR, simple sequence repeat; SCAR, sequence-characterized amplified region.

the genetic males are used for breeding (Fig. 7). It was demonstrated that the proportion of the phenotypic females in the offspring improved to about $42 \%$, representing an increase of $20 \%$ compared to that obtained with unselected male parents. This technique is now successfully applied to the artificial breeding of $C$. semilaevis and has significantly promoted commercial fish production and enhanced the financial returns.

\section{Conclusions and future perspectives}

C. semilaevis has useful traits that have been utilized for monosex breeding as well as for research on sex determination mechanisms in fish. Recent advancements in whole genome sequencing have been essential for the elucidation of the genotype and genetic foundation of phenotypic traits, and inheritance of sex determination in C. semilaevis. Epigenetic analysis has shown that heritable methylation modifications in conserved sex-related pathways and genes is crucial in the temperature induced sex reversal process. Genetic linkage maps have promoted genomic research on significant economic traits and breeding. Breakthroughs in the characterization of highly efficient sex-specific marker have provided a foundation for sex control, and have speed up the development and application of breeding techniques, such as fry production with a high proportion of females. Artificial gynogenesis techniques have also been developed and successfully induced WW super-females, which are useful for studying sex determination mechanisms and development of pure lines in C. semilaevis. However, there are still many issues 
remaining to be studied and resolved. For example, although the putative male-determining gene has been identified as $d m r t 1$, its specific function, as well as that of other sex-related genes needs to be experimentally validated. In addition, little is known about genes crucial for controlling gonad differentiation and female gonad development. Moreover, it is necessary to tighten the connection between knowledge of sex determination mechanisms and breeding practice to ensure fish production is based on the best available information. Researchers also need to meet the challenge of some key technical problems in sex control techniques, such as improving the survival rate of super-females and reducing the cost of artificial gynogenesis. Expanded applications of such technological advancements promise to increase the scale, sustainability and profitability of aquaculture of C. semilaevis.

Acknowledgements This study was supported by grants from National Natural Science Foundation of China (31130057), 'Taishan Scholar' Construction Project Special in Shandong Province, and Agricultural Improved Variety Project of Shandong Province.

Compliance with ethics guidelines Qian Zhou and Songlin Chen declare that they have no conflict of interest or financial conflicts to disclose.

This article is a review and does not contain any studies with human or animal subjects performed by any of the authors.

\section{References}

1. Liu X Z, Chen S L, Jiang Y W, Zhuang Z M, Zhai J M, Liu S T, Chen S Q, Wan RJ, Ma A J, Chang Q. Development and application of large scale breeding and healthy aquaculture technology of the half-smooth tongue sole, Cynoglossus semilaevis. China Science and Technology Achievements, 2011, (4): 10-11 (in Chinese)

2. Ji X S, Liu H W, Chen S L, Jiang Y L, Tian Y S. Growth differences and dimorphic expression of growth hormone $(\mathrm{GH})$ in female and male Cynoglossus semilaevis after male sexual maturation. Marine Genomics, 2011, 4(1): 9-16

3. Chen S L, Tian Y S, Yang J F, Shao C W, Ji X S, Zhai J M, Liao X L, Zhuang Z M, Su P Z, Xu J Y, Sha Z X, Wu P F, Wang N. Artificial gynogenesis and sex determination in half-smooth tongue sole (Cynoglossus semilaevis). Marine Biotechnology, 2009, 11(2): 243251

4. Zhuang Z M, Wu D, Zhang S C, Pang Q X, Wang C L, Wan R J. Gbanding patterns of the chromosomes of tonguefish Cynoglossus semilaevis Günther, 1873. Journal of Applied Ichthyology, 2006, 22 (5): 437-440

5. Chen S L, Zhang G J, Shao C W, Huang Q F, Liu G, Zhang P, Song W T, An N, Chalopin D, Volff J N, Hong Y, Li Q, Sha Z X, Xie M S, Yu Q L, Liu Y, Xiang H, Wang N, Wu K, Yang C G, Zhou Q, Liao X L, Yang L F, Hu Q M, Zhang J L, Meng L, Jin L J, Tian Y S, Lian J M, Yang J F, Miao G D, Liu S S, Liang Z, Yan F, Li Y Z, Sun B, Zhang H, Zhang J, Zhu Y, Du M, Zhao Y W, Manfred S, Tang Q S, Wang J. Whole-genome sequence of a flatfish provides insights into
ZW sex chromosome evolution and adaptation to a benthic lifestyle. Nature Genetics, 2014, 46(3): 253-260

6. Shao C W, Chen S L, Scheuring C F, Xu J Y, Sha Z X, Dong X L, Zhang H B. Construction of two BAC libraries from half-smooth tongue sole Cynoglossus semilaevis and identification of clones containing candidate sex-determination genes. Marine Biotechnology, 2010, 12(5): 558-568

7. Zhang J J, Shao C W, Zhang L Y, Liu K, Gao F T, Dong Z D, Xu P, Chen S L. A first generation BAC-based physical map of the halfsmooth tongue sole (Cynoglossus semilaevis) genome. BMC Genomics, 2014, 15(1): 215

8. Song W, Li Y, Zhao Y, Liu Y, Niu Y, Pang R, Miao G, Liao X, Shao C, Gao F, Chen S L. Construction of a high-density microsatellite genetic linkage map and mapping of sexual and growth-related traits in half-smooth tongue sole (Cynoglossus semilaevis). PLoS ONE, 2012, 7(12): e52097

9. Liao X L, Ma H Y, Xu G B, Shao C W, Tian Y S, Ji X S, Yang J F, Chen S L. Construction of a genetic linkage map and mapping of a female-specific DNA marker in half-smooth tongue sole (Cynoglossus semilaevis). Marine Biotechnology, 2009, 11(6): 699-709

10. Sha Z X, Luo X H, Liao X L, Wang S L, Wang Q L, Chen S L. Development and characterization of 60 novel EST-SSR markers in half-smooth tongue sole Cynoglossus semilaevis. Journal of Fish Biology, 2011, 78(1): 322-331

11. Liao X L, Shao C W, Tian Y S, Chen S L. Polymorphic dinucleotide microsatellites in tongue sole (Cynoglossus semilaevis). Molecular Ecology Notes, 2012, 66(6): 1907-1916

12. Hu Q M, Wang K L, Chen S L. Protein expression, purification, and elementary function of Dmrt1 in half-smooth tongue sole (Cynoglossus semilaevis). Journal of Fishery Sciences of China, 2013, 20 (6): 1132-1138 (in Chinese)

13. Shao C W, Li Q Y, Chen S L, Zhang P, Lian J M, Hu Q M, Sun B, Jin L J, Liu S S, Wang Z J, Zhao H, Jin Z, Liang Z, Li Y, Zheng Q, Zhang Y, Wang J, Zhang G J. Epigenetic modification and inheritance in sexual reversal of fish. Genome Research, 2014, 24 (4): 604-615

14. Dong X L, Chen S L, Ji X S, Shao C W. Molecular cloning, characterization and expression analysis of Sox $9 a$ and Foxl2 genes in half-smooth tongue sole (Cynoglossus semilaevis). Acta Oceanologica Sinica, 2011, 30(1): 68-77

15. Deng S P, Chen S L, Xu J Y, Liu B W. Molecular cloning, characterization and expression analysis of gonadal $\mathrm{P} 450$ aromatase in the half-smooth tongue-sole, Cynoglossus semilaevis. Acqaculture, 2009, 287(1-2): 211-218

16. Deng S P, Chen S L, Liu B W, Xu J Y, Tian Y S. Molecular cloning, characterization and expression analysis of brain p450arom in halfsmooth tongue-sole, Cynoglossus semilaevis Günther. Zoological Research, 2008, 29(1): 17-24 (in Chinese)

17. Liu S S, Sun B, Liang Z, Zhang J, Chen S L. Cloning and expression of anti-Müllerian hormone gene in half-smooth tongue sole, Cynoglossus semilaevis. Journal of Fishery Sciences of China, 2013, 20(1): 35-43 (in Chinese)

18. Liu W J, Zhang L Y, Shao C W, Wang N, Liu K, Wen H S, Zhang N, Dong $\mathrm{Z} \mathrm{D}$, Zhang J J, Chen S L. Molecular characterization and functional divergence of two Gadd45g homologs in sex determination in half-smooth tongue sole (Cynoglossus semilaevis). Com- 
parative Biochemistry and Physiology Part B: Biochemistry \& Molecular Biology, 2014, 177-(178): 56-64

19. Liu W J, Zhang L Y, Shao C W, Wang K L, Qi Q, Chen S L, Wen H S. Cloning and expression analysis of Gadd $45 \mathrm{~g} 3$ in half-smooth tongue sole (Cynoglossus semilaevis). Journal of Fishery Sciences of China, 2014, 21(5): 925-930 (in Chinese)

20. Zhang H, Chen S L, Wen H S, Zhu Y. WT1a gene molecular cloning and expression analysis during gender differentiation in half-smooth tongue sole (Cynoglossus semilaevis). Journal of Fishery Sciences of China, 2014, 21(1): 26-36 (in Chinese)

21. Deng S P, Chen S L, Liu B W. Molecular cloning and expression analysis of FTZ-F1 in the half-smooth tongue sole, Cynoglossus semilaevis. Zoological Research, 2008, 29(6): 592-598 (in Chinese)

22. Hu Q M, Chen S L. Cloning, genomic structure and expression analysis of ubc9 in the course of development in the half-smooth tongue sole (Cynoglossus semilaevis). Comparative Biochemistry and Physiology Part B: Biochemistry \& Molecular Biology, 2013, 165(3): 181-188

23. Chen S L, Li J, Deng S P, Tian Y S, Wang Q Y, Zhuang Z M, Sha Z $\mathrm{X}, \mathrm{Xu} \mathrm{J}$ Y. Isolation of female-specific AFLP markers and molecular identification of genetic sex in half-smooth tongue sole (Cynoglossus semilaevis). Marine Biotechnology, 2007, 9(2): 273-280

24. Chen S L, Ji X S, Shao C W, Li W L, Yang J F, Liang Z, Liao X L, $\mathrm{Xu} \mathrm{G} \mathrm{B,} \mathrm{Xu} \mathrm{Y,} \mathrm{Song} \mathrm{W} \mathrm{T.} \mathrm{Induction} \mathrm{of} \mathrm{mitogynogenetic} \mathrm{diploids}$ and identification of WW super-female using sex-specific SSR markers in half-smooth tongue sole (Cynoglossus semilaevis). Marine Biotechnology, 2012, 14(1): 120-128

25. Wang X, Zhang Q, Sun X, Yin G, Qi J, Wang Z, Wang X. Isolation and characterization of 64 novel microsatellite markers from a fosmid library of female half-smooth tongue sole (Cynoglossus semilaevis). Molecular Ecology Resources, 2008, 8(6): 13031306 